\title{
Seroprevalence and Risk Factors of Varicella-Zoster Among Children of Kashan-Center of Iran
}

\author{
Abbas Taghavi Ardakani ${ }^{1}$, Babak Soltani ${ }^{1,}$, Mojtaba Sehat ${ }^{2}$, Somaye Namjoo ${ }^{1}$ \\ ${ }^{1}$ Department of Pediatrics, Kashan University of Medical Sciences, Kashan, IR Iran \\ 2 Department of Community Medicine, Kashan University of Medical Sciences, Kashan, IR Iran \\ ${ }^{*}$ Corresponding author: Babak Soltani, Department of Pediatrics, Kashan University of Medical Sciences, Kashan, IR Iran. Tel: +98-3615575840, Fax: +98-3615550026, E-mail: babak_ \\ soltani1969@yahoo.com.
}

Received: October 01, 2012; Revised: March 15, 2013; Accepted: March 25, 2013

Background: Varicella, the primary infection of varicella-zoster virus (VZV), is a highly contagious and vaccine preventable infectious disease. It can cause severe complications in neonates and adults. Herpes zoster results from VZV reactivation later in life.

Objectives: This study was performed to determine the seroprevalence of VZV by evaluating the specific IgG antibody in 1-15 year-old children in Kashan.

Patients and Methods: This cross-sectional study was carried out on 558, one through fifteen year-old children among health-care centers in Kashan city during 2011. IgG antibodies against VZV were measured in sera by enzyme-linked immunosorbent assay (ELISA).

Results: In total, $27.6 \%$ of children were seropositive. The seroprevalence of varicella zoster antibodies increased by age (12.7\% in $1-5$ year-old, $34.4 \%$ in 6-10 year-old and 39.6\% in 11-15 year-old children). There was no difference in seroprevalence of VZV according to sex and habitation zone. By using multivariate analysis, independent factors associated with seropositivity were the family size being greater than 4 (OR= $2.01,95 \% \mathrm{CI} 1.35-3.29 \mathrm{P}=0.001)$ and history of varicella $(\mathrm{OR}=39.31,95 \% \mathrm{CI} 22.79-67.79 \mathrm{P}<0.001)$. The Negative predictive value of varicella history was $91.1 \%$ and slightly decreased by age.

Conclusions: In this region, a significant proportion of children are susceptible to VZV, severe varicella and it's complications at older ages. It is recommended for varicella vaccine to be added to routine childhood vaccination programs and also to be injected to susceptible adolescents. Negative history of varicella would be a predictor of varicella antibody status in children and young adults.

Keywords: Varicella Zoster; IgG; Seroprevalence

\section{Background}

Varicella is usually a self-limited viral illness, but in infants, adolescents, adults and immune-compromised patients, it may result in severe complications and mortality (1). Primary infection in pregnant women during the first 20 weeks of gestation may be associated with congenital infection and severe anomalies in $2 \%$ of fetuses. Infection during the third trimester and near delivery can cause severe and fatal infection in neonates. Furthermore, this virus can be latent in sensory neural ganglions and its reactivation can result in herpes zoster (2). The prevalence of varicella is different in various countries. In temperate regions without varicella vaccination, chicken pox is a childhood disease.

Many studies in Europe and America indicate that more than $90 \%$ of children are infected with varicella before 15 years old and achieve immunity against it (3-6), whereas in tropical regions, the age of varicella is older and usual- ly occurs in adolescents and adults, while it is also associated with more severity and complications (7-9). Varicella is a vaccine preventable disease. After beginning a single dose of varicella vaccine in 1995 in America, morbidity and mortality has decreased dramatically; for instance, the infection rate was reduced by $91 \%$, admission by $88 \%$ and mortality by $87 \%$ in 2005 (1).

There are similar reports for other countries after beginning varicella vaccine program (10-13). Varicella vaccination has not been incorporated in routine vaccination programs in Iran. In order to prepare the vaccination protocol, at first the varicella epidemiology must be surveyed in different climates of Iran. Few seroepidemiological studies have been conducted in Tehran and south of our country on children, with different results and no study has been performed in central part of Iran such as Kashan. Kashan district, with a population of 500 thousand, is located in a tropical desert with altitude of 982 meters from see level. The temperature of this city reach- 
es 43 degree centigrade in summers (14). In this study we have tried to survey the seroepidemiology of varicella zoster in 1-15 year- old children.

\section{Objectives}

The purpose of this study was to estimate the seroprevalence and risk factors of varicella in 1-15 year-old children in Kashan.

\section{Patients and Methods}

This cross-sectional and age-stratified study was carried out on 1-15 year-old children in Kashan. The children were chosen from the only referral pediatric hospital of the area and five public health centers randomly (cluster random sampling) to reflect the portion of the population with lower accessibility to hospital care. Study population was subdivided into three age groups, with 5-year range for each group. Weighted random selection of subjects in any age group was done according to the proportion of age groups based on the 2011 census. Children with a history of blood reception or immunoglobulin during the last year and immune-compromised patients were excluded.

A questionnaire was completed by the subject's parents, which included age, sex, family size and history of exanthematic diseases, including varicella. Sample size was determined by estimation of 30\% positive serology (6), confidence interval of $95 \%$, power of $80 \%$ and design effect of 1.5. After written informed consent was obtained, $2 \mathrm{ml}$ of blood sample was taken from each participant. Serum samples were storedat $-20^{\circ} \mathrm{C}$ until testing. Anti-VZV IgG was measured by a commercially available immunosor- bent assay (ELISA) test. The applied kit was produced by Spanish VIRCELL, S.L company with sensitivity and specificity of $96 \%$. According to the leaflet of the company, an antibody index of more than 11 was interpreted as positive, less than 9 was negative and 9-11 was indeterminate.

Statistical analysis was performed by SPSS version 17.0. The prevalence of anti-VZV antibodies for each study group was reported. T-test, chi-squared test and Fisher's exact test were used for comparison of groups and logistic regression was used for adjustment of confounding factors, considering $\mathrm{P}<0.05$ as statistically significant. We also calculated the positive predictive value (PPV $=$ proportion of people who were seropositive among those reporting a positive history of varicella) and negative predictive value (NPV = proportion of people who were seronegative among those reporting a negative history of varicella) of varicella history.

\section{Results}

In total, 569 children were studied and 11 were excluded because of receiving blood or immunoglobulin. From the 558 children, 154 (27.6\%) were positive for varicella IgG (seropositive) and 404 (72.4\%) were negative for varicella IgG (seronegative). The prevalence of anti-varicella antibody raised by increasing the age. There was a significant antibody difference between age groups (Table 1). The prevalence of antibody positivity increased by about $17 \%$ with very year of age increase. Two hundred thirty five children were male and three hundred twenty three were female. $23.4 \%$ of males and $30.7 \%$ of females were seropositive. No significant antibody positivity difference was detected according to sex and residential area (Table 1).

\begin{tabular}{|c|c|c|c|}
\hline Characteristics & Seronegative Frequency (\%) & Seropositive Frequency (\%) & Pvalue $^{a}$ \\
\hline Age, $y$ & & & $<0.0001$ \\
\hline $1-5$ & $185(87.3)$ & $27(12.7)$ & \\
\hline $6-10$ & $126(65.6)$ & $66(34.4)$ & \\
\hline $11-15$ & $93(60.4)$ & $61(39.6)$ & \\
\hline Sex & & & $<0.059$ \\
\hline Female & $180(76.6)$ & $55(23.4)$ & \\
\hline Male & $224(69.3)$ & $99(30.7)$ & \\
\hline Family members & & & $<0.001$ \\
\hline$\leq 4$ & $339(76.9)$ & $102(23.1)$ & \\
\hline$>4$ & $65(55.6)$ & $52(44.4)$ & \\
\hline Place of Life & & & $<0.059$ \\
\hline Urban & $345(71.3)$ & $139(28.7)$ & \\
\hline Rural & $59(79.7)$ & $15(20.3)$ & \\
\hline
\end{tabular}

a Pearson Chi-Square

Children who live in families of $>4$ had seropositivity, 2.10 times more than children with family size $\leq 4$, af- ter adjustment for sex and age $(\mathrm{OR}=2.01,95 \% \mathrm{CI} 1.35-3.29$ $\mathrm{P}=0.001)$. Eighty one percent of those with a history of 
chicken pox were seropositive while $9 \%$ of those without a history of chicken pox were seropositive. Children with a history of chicken pox had seropositivity of 39.3 times greater than children without this history $(\mathrm{OR}=39.31,95 \%$ CI 22.79 - 67.79, $\mathrm{P}<0.001)$. The correlation between age and seropositivity percent was $0.85(\mathrm{P}<0.001)$ (Figure 1$)$.

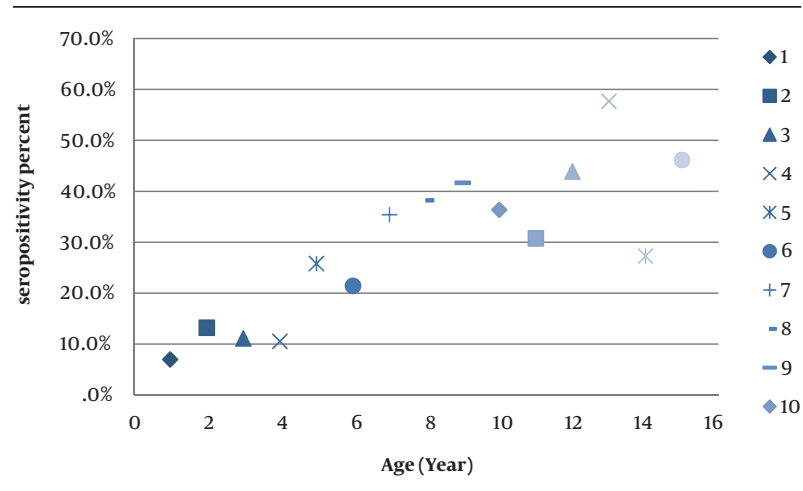

Figure 1. Correlation Between age and Seropostive Proportion of Varicella-zoster Virus (VZV) in Children 1-15 Years

According to Figure 2, the positive predictive value of varicella history was $81.2 \%$ and negative predictive value was $91.1 \%$.

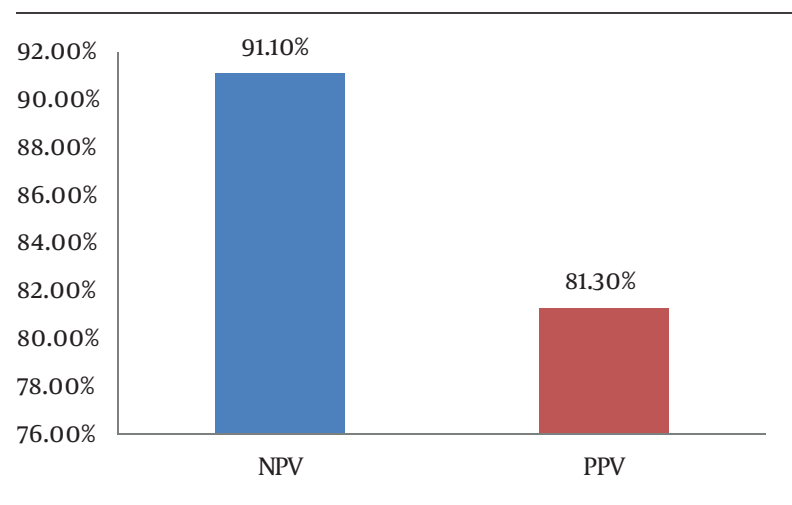

Figure 2. PPV and NPV of Varicella Infection History

\section{Discussion}

Varicella is a vaccine preventable disease. For preparation of vaccine protocol, varicella epidemiology should be evaluated in all parts of the country. The accessibility and safety of varicella vaccine, has favored the epidemiologic studies in the world. As the infection with this virus is highly contagious and usually induces lifelong immunity, the measurement of serum specific varicella IgG is an acceptable tool for detection of previous infection (2). In this study, $27.6 \%$ of 558 children were seropositive. With increase of age, the seroprevalence raised by $12.7 \%$ in $1-5$ year-old, $34.4 \%$ in 6-10 year-old and 39.6\% in $11-15$ yearold children, which was lower than our expectation. In Ehsanipour's study in Tehran (2005), seroprevalence of
Varicella in Children, one through sixteen year-old referring to Hazrat-e- Rasool Akram Hospital was 42.5\% (15). In another study by Sharifi et al. in Tehran/Iran on 635 people, the seroprevalence was positive in $59.7 \%$ of less than 10 year-old, $60.4 \%$ of $10-14$ year-old and $87.5 \%$ of $15-19$ yearold cases (16).

The seroprevalence of varicella in our research is lower than the two studies performed in Tehran. In Motamedifar's study in Shiraz/Iran (2003) on 270, six through ten year-old elementary school children, the serum varicella IgG was positive in $35.2 \%$ (13). Ziyaeyan et al. in Shiraz Namazi hospital, evaluated varicella serology in 843 one through seventy year-old people. It was positive in $25.3 \%$ of $1-5$ year-old, $43.1 \%$ of $6-10$ year-old, $73.5 \%$ of $11-15$ year-old and $86 \%$ of adults (17).Our results are closer to the above studies in Shiraz, because Kashan and Shiraz belong to tropical climates. In a study in Jahrom/Iran (2010) on child bearing aged women before marriage, one fourth of them were seronegative against varicella (18).

The varicella seroprevalence in 1-15 year olds was $37.4 \%$ in Pakistan (7), 52.3\% in Thailand (9) and 10\% in western India (8). In United Arab Emirates, $45.8 \%$ of children before 10 years old and $68.4 \%$ of $11-20$ year-old individuals were seropositive(19). In Singapore, less than one fifth of children under 15 years old and less than one half of 15-24 year-old individuals were seropositive (8). As we expected, the varicella seroepidemiology in Kashan is in accordance with tropical areas. Unlike tropical areas, in temperate regions, most unvaccinated children less than 15 years old have been infected with chicken pox and become immune against it.

There are many investigations in Europe, America and Japan (before varicella vaccination) that confirm this subject. For instance in Netherland, 93\% of children below 5(3), in Germany 94.2\% below 10 (4), in Switzerland 96.1\% below 12 (5), in Turkey 85\% below 10 (6) and in Japan 83\% below 9 years old have become seropositive (20); in these countries approximately $100 \%$ of adults have varicella antibody. Regarding the high temperature in Kashan during summer (43 degree Celsius) (14) and the varicella seroprevalence in this study and other researches in Iran and tropical regions such as Pakistan (7), it seems that the effect of temperature is more than humidity.

Our results indicate that by increasing the age, varicella immunity increases and this is compatible with other investigations. Risk factors that we studied in this survey were sex, number of family members and area of residence. There is no significant relationship between varicella seroprevalence, sex and area of residence and this is consistent with some investigations in Iran $(13,16,17)$. Our study shows a meaningful relationship between varicella serology and family members, so by increasing a person, seropositivity gets up 57\%. A Turkish survey in 2004 (21) indicated this relationship but many other reports havenot done so (6-8).

In Ehsanipour's study in Tehran there was no relation- 
ship between varicella seroposetivity and socioeconomic level, while family number was one of the items of socioeconomic status (15). Other studies in Iran didn't investigate this item. In this investigation, children with a history of chicken pox were $81.2 \%$ seropositive and $18.8 \%$ seronegative. The reason can be the similarity of varicella vesicular rash to other causes such as herpes simplex, enteroviruses, monkey pox, rickettsial pox or Staphylococcus aureus and also drug reactions can be wrongly considered as varicella rash (1). On the other hand, 8.9\% of children without any history of varicella were seropositive, which means they had mild chicken pox (1), so there was a strong relationship between seropositivity and history of varicella(positive predictive value of $81.2 \%$ and negative predictive value of $91.1 \%$ ). Based on these results, about $70 \%$ of children under 15 years old are not immune against varicella.

With regards to circulation of wild virus in the community, any of these sensitive people can be infected with varicella at older ages, which can cause them severe complications such as pneumonia and also infection of this virus can be a threat to child bearing women. Unfortunately, there are no reports of congenital varicella syndrome, TORCH and other congenital defects in this region, but with attention to the findings of this study, there is still the possibility of congenital varicella complications. In conclusion, this study states the sensitivity of a noticeable number of adolescents and adults to varicella virus. In this condition, varicella vaccine can mitigate the circulation of wild virus in the community and decrease the possibility of infection in all age groups (1).

An exact strategy should be implemented for varicella vaccine in all countries. Childhood vaccination is beneficial in temperate countries where contagion of varicella is high, but it should be done with appropriate coverage. If in these countries the varicella vaccine coverage is not complete, severe varicella will increase in older ages. World Health Organization recommends varicella vaccine only in countries with high coverage (over 90\%). The MMR (measles, mumps, rubella) coverage can represent the ability of nations for coverage of varicella vaccination in the future (22). At present, the MMR coverage in Iran is $92 \%(23)$. In countries with low prevalence of varicella such as Iran, catch of campaign is required besides childhood vaccination. Based on high negative predictive value of varicella history in this study, varicella vaccination may be more beneficial in adolescents, child bearing women and medical personnel without history of varicella. We suggest that more comprehensive studies with extended results be done to determine varicella vaccination strategy in Iran.

\section{Acknowledgements}

We are grateful to local and province public health-care centers for their cooperation.

\section{Authors' Contribution}

Taghavi Ardakani A and Soltani B did the design and analysis of the study. Sehat M performed the statistical analysis. Namjoo S provided the study subjects and data collections.

\section{Financial Disclosure}

The authors declare no financial conflicts of interest relevant to this manuscript.

\section{Funding Disclosure}

Kashan University of Medical Sciences financially supported this study.

\section{References}

1. Kligman RM, Stanton BF, Geme ST. Nelson Textbook of Pediatrics. 2011.

2. Pickering LK, Baker CJ, Kimberling DW, Long SS. Red book®:2009 Report of the committee on infectious diseases. 2009.

3. de Melker H, Berbers G, Hahne S, Rumke H, van den Hof S, de Wit A, et al. The epidemiology of varicella and herpes zoster in The Netherlands: implications for varicella zoster virus vaccination. Vaccine. 2006;24(18):3946-52.

4. Wutzler P, Farber I, Wagenpfeil S, Bisanz H, Tischer A. Seroprevalence of varicella-zoster virus in the German population. Vaccine. 2001;20(1-2):121-4.

5. Aebi C, Fischer K, Gorgievski M, Matter L, Muhlemann K. Agespecific seroprevalence to varicella-zoster virus: study in Swiss children and analysis of European data. Vaccine. 2001;19(23 24):3097-103.

6. Kanra G, Tezcan S, Badur S. Varicella seroprevalence in a random sample of the Turkish population. Vaccine. 2002;20(9-10):1425-8.

7. Akram DS, Qureshi H, Mahmud A, Khan AA, Kundi Z, Shafi S, et al. Seroepidemiology of varicella-zoster in Pakistan. Southeast Asian JTrop Med Public Health. 2000;31(4):646-9.

8. Lee BW. Review of varicella zoster seroepidemiology in India and Southeast Asia. Trop Med Int Health. 1998;3(11):886-90.

9. Migasena Sricharoen, Simasathien Sriluck, Desakorn Varunee, Phonrat Benjaluck, Suntharasamai Pravan, Pitisuttitham Punnee, et al. Seroprevalence of varicella-zoster virus antibody in Thailand. Int $j$ inf dis. 1997;2(1):26-30.

10. Asano Y. Clinicopathologic understanding and control of vari cella-zoster virus infection. Vaccine. 2008;26(50):6487-90.

11. Tanuseputro P, Zagorski B, Chan KJ, Kwong JC. Population-based incidence of herpes zoster after introduction of a publicly funded varicella vaccination program. Vaccine. 2011;29(47):8580-4.

12. Carville KS, Riddell MA, Kelly HA. A decline in varicella but an uncertain impact on zoster following varicella vaccination in Victoria, Australia. Vaccine. 2010;28(13):2532-8.

13. Motamedifar Mohammad, Handjani Farhad, Hadi Nahal, Shahkarami Mohammad Kazem, Mehrabani Davoud. Seroprevalence of Varicella-Zoster Virus in Children from Shiraz Iran. Iran J Immunol. 2006;3(1):43-6.

14. Director of Meteorology Bureau of Kashan, Kashan meteorological annual report. 2012; Available from: Available from: www. kashanmet.ir.

15. Ehsanipour F, Shayanfar N, Salarian K. Surveying of Protective Antibody against Varicella zoster Virus (VZV) Infection in Children Referring to Hazrat-e-Rasool Akram Hospital (2005). J Iran Med Sci. 2009.

16. Sharifi Z, Emadi Ghanjin S. The Seroepidemiology of Varicella Zoster Virus (VZV) in Different Age Groups in Tehran, Iran. Iran J Allergy Asthma Immunol. 2005;4(2):95-8.

17. Ziyaeyan M, Alborzi A, Jamalidoust M, Moieni M, Pourabbas B Seroepidemiology of varicella zoster virus infection among 1-70 year individuals in Iran. Iran Red Crescent Med J. 2010;12(2):176- 
180.

18. Pourahmad M, Davami MH, Jahromi AR. Evaluation of anti-varicella antibody in young women before their marriage: A seroepidemiologic study in Iran. J Clin Virol. 2010;48(4):260-3.

19. Uduman SA, Tahira AM, Al-Wash R, Usmani MA, Bener A. Varicella susceptibility among children and healthy adults in the United Arab Emirates. East Mediterr Health J. 2001;7(4-5):604-8.

20. Ueno-Yamamoto K, Tanaka-Taya K, Satoh H, Araki K, Kaku M, Okabe N. THE changing seroepidemiology of varicella in Japan: 1977-1981 and 2001-20 05. Pediatr Infect Dis J. 2010;29(7):667-9.

21. Savas S, Dallar Y, Arikan I, Onde U. [Varicella-zoster virus seroprevalence in children between 0-15 years old]. Mikrobiyol Bul. 2004;38(1-2):69-75.

22. Nardone A, de Ory F, Carton M, Cohen D, van Damme P, David- kin I, et al. The comparative sero-epidemiology of varicella zoster virus in 11 countries in the European region. Vaccine. 2007;25(45):7866-72.

23. Director of the Center for Vaccine-preventable diseases Iran CDC. Vaccination coverage in Iran. 2011; Available from: Available from: http://webda.behdasht.gov.ir/ index.aspx?siteid=326\&pag eid=32628\&newsview $=52194$.

Please cite this paper as: Taghavi Ardakani A, Soltani B, Sehat M, Namjoo S.Seroprevalence and Risk Factors of Varicella-Zoster Among Children in Kashan - Center of Iran. Jundishapur J Microbiol. 2013; 6(5): e8388. DOI: 10.5812/jjm.8388 